\title{
Correlation between Schizas Score, Degree of Disability, and Neurogenic Claudication in Lumbar Spinal Stenosis
}

\author{
Arief Indra Perdana Prasetya'), Fajar Baskoro Gardjito'), R. Andhi Prijosedjati'), \\ Pamudji Utomo'), Handry Tri Handojo²) \\ ${ }^{1)}$ Department of Orthopaedic and Traumatology, Faculty of Medicine, Universitas Sebelas Maret / \\ Dr. Moewardi Hospital/ Prof.Dr.R.Soeharso Orthopaedic Hospital \\ 2)Department of Radiology, Prof.Dr.R.Soeharso Orthopaedic Hospital, Surakarta
}

\begin{abstract}
Background: Magnetic Ressonance Imaging (MRI) is one of modality to diagnosed lumbar spinal stenosis (LSS) however there were some mismatch between the degree of canal stenosis to functional score of LSS. There are some methods to measure the degree of canal stenosis, on of them is Schizas Score. To asses functional score of patients with LSS, there are some tools can be used. Oswesstry Disability Index (ODI) and Neurogenic Claudication Outcome Score (NCOS) are questionnaire commonly used in LSS. This study aimed to analyze the correlation between the degree of stenosis grading using Schizas Score to the degree of disability using ODI score and Neurogenic Claudication using NCOS.

Subjects and Method: This was an analytic observational study conducted at Prof. Dr. R. Soeharso Orthopaedic Hospital, Surakarta, Central Java. A sample of 35 patients with LSS in were assessed by filling ODI and NCOS questionnaire, then evaluate the lumbar axial MRI to identify the Schizas Score. The data were analyzed by Spearman test.

Results: There was a significant correlation between Schizas Score to ODI score and NCOS. Schizas score increased ODI score $(r=0.91 ; \mathrm{p}<0.001)$. Schizas score decreased NCOS $(r=-0.90$; $\mathrm{p}<0.001$ ).
\end{abstract}

Conclusion: Schizas score is correlated with ODI score and NCOS.

Keywords: lumbar spinal stenosis, Schizas score, ODI, NCOS

\section{Correspondence:}

Arief Indra Perdana Prasetya. Resident of Orthopaedic and Traumatology Specialist Program, Universitas Sebelas Maret. Jl. Ir. Sutami 36A, Surakarta 57126, Central Java. Email: absolutellydarkknight@gmail.com.

\section{BACKGROUND}

Lumbar spinal stenosis (LSS) is a spinal disorder that often occurs especially in the elderly with clinical syndromes that could be presented as buttock pain or radiating to the lower extremities, with or without lower back pain and the presence of imaging features associated with narrowing of the space around the nerve and blood vessels in the lumbar vertebrae. The components of the vertebral canal that contribute to the symptoms of stenosis include facets (hypertrophy, arthropathy), yellow ligaments (hypertrophy), posterior longitudinal liga- ment (ossification of the posterior longitudinal ligament [OPLL]), vertebral body (osteophyte), intervertebral discs, and epidural fat (Fortinet al., 2017; Leeet al., 2015; Wu et al., 2017).

According to Kirkaldy-Willis's theory, the spine is a complex tripod (three-joint complex), where the degenerative cascade process begins with the occurrence of circumferential tear of the annulus which is radially burdensome. Biochemical changes in the disc cause more severe degeneration and loss of disc height. Changing of the structure and decreasing height of the disc 
triggers the protrusion of disc bulging and posterior longitudinale ligaments, so that the spinal canal getting narrow. Shortening of the disc also triggers folds in the yellow ligaments and lead to further facet joint damage. Damaged facet joints will form osteophytes, causing stenosis to the thecal sac in the spinal canal or nerve fibers in the intervertebrale foramen. Hypertrophy of the ligaments and soft tissue structures around the spinal cord can cause spinal stenosis. In this case, at the extension position, the ligament folds to the center of spinal canal so that it can aggravate the canal stenosis. Changes in structure, movement and biomechanics trigger a cycle of degeneration(Kobayashi, 2014; Patel and Truumees, 2011).

The exact prevalence of LSS is still unknown, but it is estimated that more than 200,000 adults in the United States suffer from LSS and are expected to increase to 64 million elderly adults by 2025. A study in Japan stated that the incidence of LSS increased by age, around $2 \%$ in the population of the age of 4 years, and increased between $10 \%-11 \%$ in the population aged 70-79 years. In other studies, it was stated that the prevalence of lumbar stenosis is around one tenth of the total population(Ombregt, 2013; Wu et al., 2017).

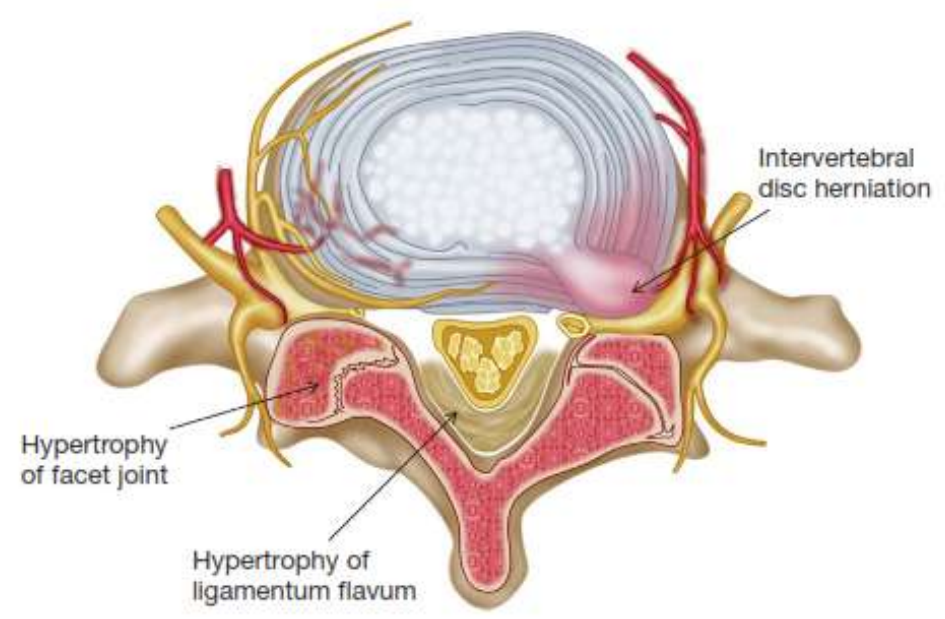

Figure 1. Canal Stenosis (Wu et al., 2017)

MRI is a non-invasive examination to evaluate the anatomy of the spine, especially the soft tissue structure in the case of lumbar stenosis. However, a mismatch between MRI images and clinical symptoms has been found where there are correlations of MRI findings and clinical findings that are not always significant. The sensitivity and specificity of MRI to assess LSS were reported to be higher than CT scans and myelography, so MRI was considered more effective in diagnosing lumbar stenosis (Ambade and Mane, 2016; Moojen et al., 2015).
The degree of lumbar stenosis can be classified based on canal images on axial MRI pieces by measuring the dural sac cross-sectional area (DSCA). Currently a new method is found as an alternative to determine the degree of stenosis based on the morphological features of lumbar axial MRI introduced by Schizas. Schizas divides the degree of stenosis based on axial MRI morphological features that assess the distribution of Cerebro-Spinal Liquor (CSF), root, epidural fat, and posterior arch (Ambade and Mane, 2016; Lonne et al., 2014; Moojen et al., 2015). 
Indonesian Journal of Medicine (2019), 4(2): 116-121

https://doi.org/10.26911/theijmed.2019.04.02.05

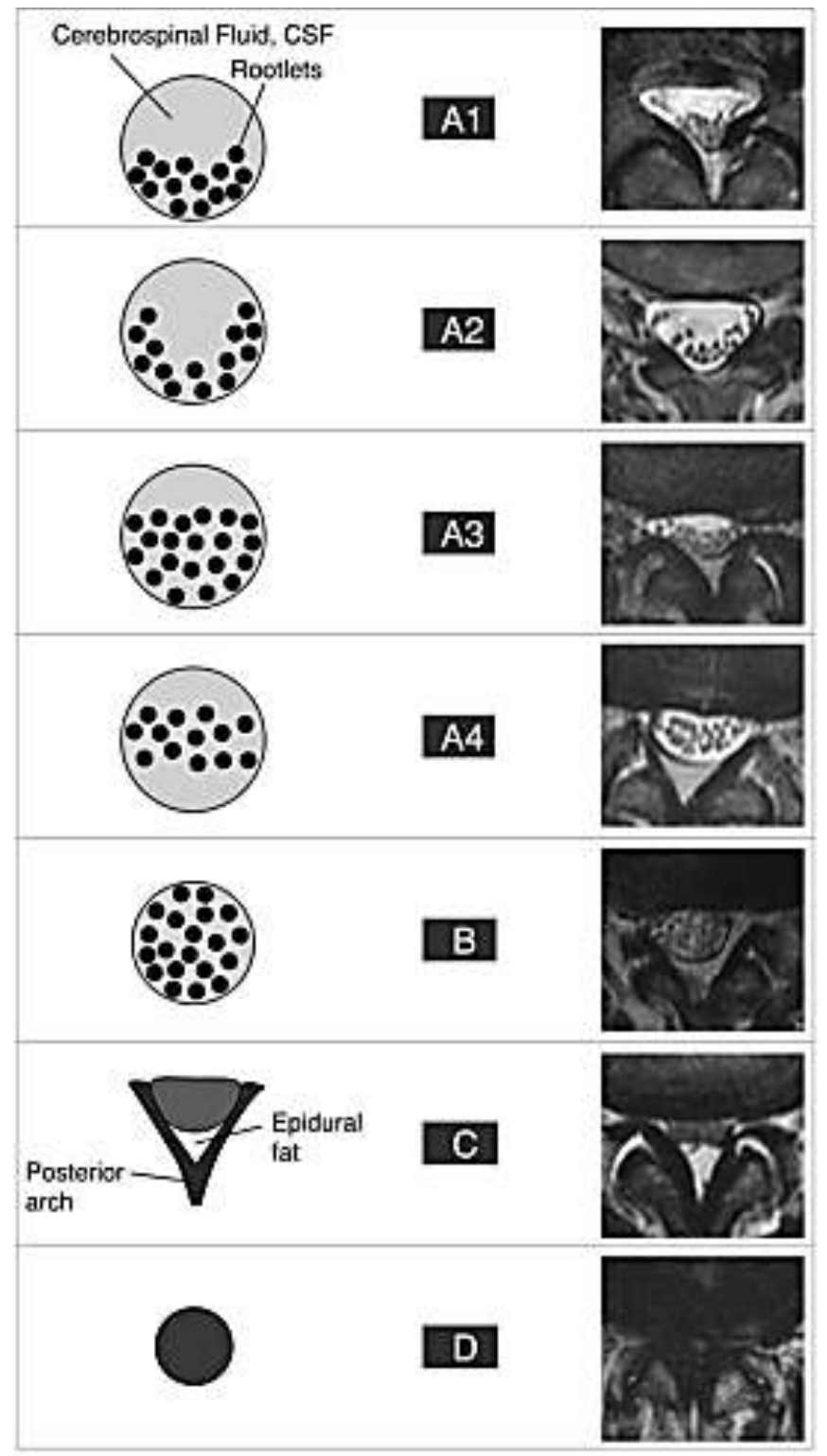

Figure 2. Degree of stenosis based on the Schizas Classification (Moojen et al., 2015)

Schizas' description of LSS on lumbar axial MRI is based on the morphological appearance of the dural sac as seen in the axial T2 image of the lumbar region's spine, taking into account the cerebrospinal fluid (CSF) content and root distribution. This grading is based on CSF ratio and root distribution seen in axial T2 MRI images by observing the pattern of root distribution in the dural sac. The description of the assessment is as follows, Grade A: CSF which is clearly visible in the dural sac, but its distribution is not homogeneous. Grade B: root occupies the entire dural sac, and can still be clearly identified. There is a picture of CSF that is still visible, but with a rough distribution of the dural sac. Grade C: there is no root that can be recognized, the dural sac shows a homogeneous gray signal without a clear picture of CSF. Epidural fat is found at the posterior site. Grade D: besides no recognizable roots, there is also no epidural fat posteriorly. Generally it can be concluded that grade A as mild, grade B 
as moderate, grade $\mathrm{C}$ as severe, and grade $\mathrm{D}$ as very severe stenosis (Ambade and Mane, 2016; Moojen et al., 2015).

The Oswestry Disability Index (ODI) is an interview-based instrument that is generally accepted to measure disability in patients with lumbar spinal stenosis (LSS). ODI evaluates the disruption of general activities of daily life due to lower back pain and assesses the quantity of patient complaints to determine their degree of disability. ODI consists of ten questions and is assessed in percentage, with interpretation: Mild (o\% to $20 \%$ ); moderate (21\% to $40 \%$ ); severe (41\% to $60 \%$ ); paralyzed (61\% to $80 \%$ ); and bedridden ( $81 \%$ to $100 \%$ ) (Adamova et al., 2013; Goni et al., 2014).

Neurogenic Claudication Outcome Score (NCOS) is a simple, concise questionnaire, and specifically adapted to assess function in patients with symptoms of neurogenic claudication. Each item is rated with a four-point scale with two intervals ranging from 0 to 6 (0-2-4-6), where the value 0 indicates the worst condition to the value 6 which shows the best results. Pain intensity is added by calculating the Visual Analog Scale (VAS), where the patient chooses a point on the line representing the perception of pain intensity. The score calculated by summing all items with the lowest value 0 to a maximum score of 100 , where higher scores indicate higher levels, and are interpreted as better functional status (Azimi et al., 2015; Suyasa et al., 2018).

There is still any mismatched between the MRI features and the clinical condition of the patient, and there is still no research that analyze the correlation between the degree of stenosis based on Schizas Score and the degree of disability and neurogenic claudication experienced by patients, especially in Lumbar Spinal Stenosis patients in Indonesia. This study aimed to determine the correlation between the degree of stenosis in axial MRI images using the Schizas Score with quality of life and the degree of neurogenic claudication in patients with lumbar stenosis.

\section{SUBJECTS AND METHOD \\ 1. Study Design \\ This was an analytic observational study conducted at Prof. Dr.R.Soeharso Ortho- paedic Hospital, Surakarta.}

\section{Population and Sample}

The study population was all patients treated at Prof. Dr. R. Soeharso Orthopaedic Hospital with lumbar stenosis. Samples were selected from these patients by simple random sampling that met the inclusion criteria, which are age $\geq 40$ years and had complaints of lumbar stenosis at least 1 month, with no history of trauma, infection, tumor, or other neuromuscular abnormalities of the spine. There was 35 samples in total.

\section{Study Variables}

The dependent variables were ODI and NCOS scores. The independent variable was Schizas score.

\section{Study instrument}

Data on lumbar MRI was obtained from medical record in Radiology Department, Prof. Dr. R Soeharso Orthopaedic Hospital, Surakarta. Schizas score was assessed by axial T2 MRI image. Disability degree and neurogenic claudication were collected by ODI and NCOS questionnaire.

\section{Data Analysis}

The data were analyzed by Spearman nonparametric test to determine the relationship between Schizas Score and the quality of life and neurogenic claudication.

\section{RESULTS}

1. Sample characteristics

From the results of collecting samples of patients with lumbar spinal stenosis, based 
on the age distribution,the youngest age of 41 years and the oldest 78 years.As for when grouped based on the range of decades of age, data obtained from patients aged 40-50 years old as many as 6 people (17.1\%), 51-60 years old as many as 16 people (45.7\%), 61-70 years old as many as 11 people (31.4\%), and two people who are over 70 years old.
From the distribution of patients based on sex, there were 14 male patients and 21 female. Based on the distribution of patient data, the number of lumbar stenosis patients with Schizas A stenosis levels was 11 people (31.4\%), Schizas B and C as many as 7 people (20.0\%), and Schizas D as many as 10 people (28.6\%). Data distribution of lumbar stenosis patients was presented in Table 1.

Table 1. Data Distribution of Lumbar Stenosis Patients

\begin{tabular}{lcc}
\hline Characteristics & n & \% \\
\hline Age & 6 & $17.1 \%$ \\
$40-50$ years old & 16 & $45.7 \%$ \\
$51-60$ years old & 11 & $31.4 \%$ \\
61-70 years old & 22 & $5.8 \%$ \\
770 years old & & \\
Sex & 14 & $40.0 \%$ \\
Male & 21 & $60.0 \%$ \\
Female & & \\
Stenosis degree (Schizas Score) & 11 & $31.4 \%$ \\
A & 7 & $20.0 \%$ \\
B & 7 & $20.0 \%$ \\
C & 10 & $28.6 \%$ \\
D & & \\
\hline
\end{tabular}

\section{Bivariate analysis}

The results of Spearman test was described in Table 2. There was a significant correlation between Schizas Score to ODI score

Table 2. Correlation Analysis Between Schizas Score to ODI Score and NCOS

\begin{tabular}{lccc}
\hline \multicolumn{1}{c}{ Correlation } & $\mathbf{n}$ & $\mathbf{r}$ & P \\
\hline Correlation between Schizas Score and ODI Score & 35 & 0.91 & $<0.001$ \\
Correlation between Schizas Score and NCOS & 35 & -0.90 & $<0.001$ \\
\hline
\end{tabular}

\section{DISCUSSION}

Based on patients distribution data of this study, lumbar stenosis starts at age of 40 years old and increased with age. From previous studies, it was found that for ages of fourth until fifth decades, stenosis symptoms were $1.7-2.2 \%$ and the prevalence was increased by age, which is at the sixth to seventh decades can reach almost 50\%. From the sex distribution of data obtained in this study is same with the prevalence study that lumbar spinal stenosis patients and NCOS. Schizas score increased ODI score $(\mathrm{r}=0.91 ; \mathrm{p}<0.001)$. Schizas score decreased NCOS $(r=-0.90 ; p<0.001)$. 
Schizas Score and NCOS. Higher degree of stenosis decreased NCOS.

\begin{tabular}{c}
\hline REFERENCES \\
\hline Adamova BM, Vohanka S, Hnojcikova B, \\
Okacova I, Dusek L, Bednarik J \\
(2013). Neurological impairment \\
score in lumbar spinal stenosis. Eur \\
Spine J, 22(8): 1897-1906.
\end{tabular}

Ambade RE, Mane KK (2016). Clinical evaluation of lumbar spinal stenosis and its correlation with the MRI. Indian J Orthop Surg, 2(3), 293-305.

Azimi P, Mohammadi HR, Benzel EC, Shahzadi S, Azhari S (2015). Lumbar spinal canal stenosis calssification criteria: a new tool. Asian Spine J, 9(3): 399-406.

Cláudio L, Rodrigues L (2016). Lumbar Spinal Stenosis, Clinical Presentation, Diagnosis, and Treatment. In From bench to bedside - trauma, tumors, spine, functional neurosurgery $2: 83^{-}$ 97). InTech.

Fortin M, Lazary A, Varga PP, Battle MC (2017). Association between paraspinal muscle morphology, clinical symptoms and functional status in patients with lumbar spinal stenosis. Eur Spine J. 26(10): 2543-2551.

Goni VG, Hampannavar A, Gopinathan NR, Singh P, Sudesh P, Logithasan RK et al. (2014). Comparison of the oswestry disability index and magnetic renonance imaging findings in lumbar canal stenosis: an observational study. Asian Spine J. 8(1): 44-50.

Kobayashi S (2014). Pathophysiology, diagnosis and treatment of intermittent claudication in patients with lumbar canal stenosis. World J Orthop. 5(2): 134-145.
Lee SY, Kim TH, Oh JK, Lee SJ, Park MS (2015). Lumbar stenosis: a recent update by review of literature. Asian Spine J. 9(5): 818-828.

Lonne G, Odegard B, Johnsen LG, Solberg TK, Kvistad KA, Nygaard OP (2014). MRI evaluation of lumbar spinal stenosis: is a rapid visual assessment as good as area measurement? Eur Spine J. 23(6): 1320-1324.

Moojen WA, Schenck CD, Lycklama NG, Jacobs WCH, Van der Kallen BF, et al. (2015). Preoperative MR imaging in patients with intermittent neurogenic claudication: relevance for diagnosis and prognosis. Spine. 43(5): 348-355.

Ombregt L (2013). Applied anatomy of the lumbar spine. In A system of orthopaedic medicine (3rd ed.): 415-436. Edinburgh: Churchill Livingstone Elsevier.

Patel CK, Truumees E (2011). Spinal stenosis: pathophysiology, clinical diagnosis, and differential diagnosis. In $\mathrm{HN}$ Herkowitz, SR Garfin, FJ Eismont, GR Bell, RA Balderston (Eds.), Rothman-simeone the spine: expert consult (vol. 2) (6th ed.): 1064-1077. Philadelphia: Elsevier Saunders.

Suyasa IK, Adiwardhana IGS, Setiawan IGY (2018). A comparison of neurogenic claudication outcome score and oswestry disability index in degenerative lumbar spinal canal stenosis post decompression, posterior stabilization and fusion. Int $\mathrm{J}$ Orthop Sci, 4(2), 409-411.

Wu A, Zou F, Cao Y, Xia D, He W, Zhu B, et al. (2017). Lumbar spinal stenosis: an update on the epidemiology, diagnosis and treatment. AME Medical Journal, 2(63): 1-14. 